Methods Virally suppressed patients (HBV DNA $<20 \mathrm{IU} / \mathrm{mL}$ at screening) on TDF were randomized $(1: 1)$ to switch to TAF or continue TDF for 48 weeks in a double-blind fashion. Viral suppression and changes in bone (BMD by DXA) and renal (creatinine clearance $\left[\mathrm{eGFR}_{\mathrm{CG}}\right]$ ) parameters were assessed over 48 weeks.

Results Among the 400 Asian patients enrolled, 288 (72\%) had at least 1 TDF RF. At Week 48, similar proportions with $\geq 1 \mathrm{RF}$ had HBV-DNA <20IU/mL (TAF 97\%; TDF 97\%) and normal ALT by 2018 AASLD criteria (TAF 76\%; TDF 73\%). TAF subjects with $\geq 1 \mathrm{RF}$ had increases in eGFR $_{\mathrm{CG}}$ compared to decreases on TDF [median $(\mathrm{Q} 1, \mathrm{Q} 3)$ change; TAF: + 2.6 (2.01, 7.34); TDF: -2.7 (-7.56, +15.79); p<0.0001)]. Among patients with $\geq 1 \mathrm{RF}$, improvements were seen in BMD for TAF vs. continued declines in TDF patients at both spine $(\mathrm{p}<0.0001)$ and hip $(\mathrm{p}<0.0001)$.

Conclusions Virally suppressed Asian patients with $\mathrm{CHB}$ and risk factors for TDF who switched to TAF showed improved bone and renal safety while efficacy was well-maintained.

\section{IDDF2020-ABS-0059 SAFETY AND EFFICACY OF SOFOSBUVIR/ VELPATASVIR (SOF/VEL) IN PEDIATRIC PATIENTS 6 TO $<18$ YEARS OLD WITH CHRONIC HEPATITIS C (CHC) INFECTION}

\begin{abstract}
${ }^{1}$ Maureen Jonas*, ${ }^{2}$ Rene Romero, ${ }^{3}$ Etienne Sokal, ${ }^{4}$ Philip Rosenthal, ${ }^{5}$ Gabriella Verucchi, ${ }^{6}$ Chuan-Hao Lin, ${ }^{7}$ Jessica Wen, ${ }^{8}$ Michael Narkewicz, ${ }^{9}$ Sanjay Bansal, ${ }^{10}$ Jiang Shao, ${ }^{10}$ Sean Hsueh, ${ }^{10}$ Anuj Gaggar, ${ }^{10}$ Kathryn Kersey, ${ }^{10}$ Carol Yee Kwan Chan, ${ }^{11}$ Regino Gonzalez-Peralta, ${ }^{12}$ Daniel Leung, ${ }^{13}$ William Balistreri, ${ }^{14}$ Karen Murray, ${ }^{15}$ Kathleen Schwarz. ${ }^{1}$ Boston Children's Hospital, Boston, MA, USA; ${ }^{2}$ Emory University School of Medicine and Children's Healthcare of Atlanta, Atlanta, USA; ${ }^{3}$ Cliniques Universitaires Saint-Luc, UC Louvain, Brussels, Belgium; ${ }^{4}$ University of California San Francisco, San Francisco, USA; ${ }^{5}$ University of Bologna, Bologna, Italy; ${ }^{6}$ Children's Hospital Los Angeles, Los Angeles, USA; 'University of Pennsylvania and The Children's Hospital of Philadelphia, Pennsylvania, USA; ${ }^{8}$ University of Colorado School of Medicine and Children's Hospital of Colorado, Aurora, USA; ${ }^{9}$ Kings College Hospital, UK; ${ }^{10}$ Gilead Sciences, InC, Foster City, USA; ${ }^{11}$ Pediatric Gastroenterology, Hepatology and Liver Transplant, AdventHealth for Children, Orlando, USA; ${ }^{12}$ Baylor College of Medicine and Texas Children's Hospital, Houston, USA; ${ }^{13}$ Cincinnati Children's Hospital Medical Center, Cincinnati, USA; ${ }^{14}$ University of Washington School of Medicine and Seattle Children's Hospital, Seattle, USA; ${ }^{15}$ Johns Hopkins University School of Medicine, Baltimore, USA
\end{abstract}

\subsection{6/gutjnl-2020-IDDF.142}

Background DAA regimens have been approved for $\mathrm{CHC}$ treatment in 12 to $<18$ years, but for younger children, the standard of care is still pegylated-interferon plus ribavirin for $\leq 48$ weeks. We evaluated the safety and efficacy of the pangenotypic regimen of SOF/VEL in children 6 to $<18$ years.

Methods Patients 6 to $<18$ years with $\mathrm{CHC}$ of any genotype (GT) were enrolled in this open-label, ongoing study. Patients 6 to $<12$ years received SOF/VEL $200 \mathrm{mg} / 50 \mathrm{mg}$ and 12 to $<18$ years SOF/VEL $400 \mathrm{mg} / 100 \mathrm{mg}$ QD for 12 weeks. Key efficacy endpoint was SVR12. Safety was assessed by adverse events (AEs) and clinical/laboratory data. Intensive pharmacokinetic sampling on Day 7 in a subgroup of patients of each age group was done to confirm the appropriateness of the chosen dose.

Results 102 patients 12 to $<18$ years and 73 patients 6 to $<12$ years were enrolled. GT distribution was 75\% GT1, 5\% GT2, 13\% GT3, 3\% GT4, 3\% GT6; 51\% female, 80\% white, $85 \%$ treatment naïve, and $91 \%$ vertically infected. Intensive pharmacokinetics confirmed that the doses selected were appropriate. SVR12 rate was 95\% (97/102) and 92\% (67/73) among patients 12 to $<18$ years and 6 to 12 years respectively; 1 patient in each age group had virologic failure, the remaining patients did not achieve SVR for non-virologic reasons. Most AEs were mild or moderate. 5 subjects had serious $\mathrm{AE}$ none of which was attributed to treatment, 2 patients discontinued treatment due to AEs and considered treatment unrelated. The most common AEs (>15\%) were headache, fatigue, and nausea in adolescents and vomiting, cough and headache in 6 to $<12$ years.

Conclusions In patients 6 to $<18$ years with GT1, 2, 3, 4 or $6 \mathrm{CHC}$ infection, treatment with SOF/VEL for 12 weeks resulted in $\geq 92 \%$ SVR12 rate. SOF/VEL was well tolerated, supporting its potential as a treatment option for children 6 to 17 years of age. The study is ongoing in children aged 3 to $<6$ years old.

\section{IDDF2020-ABS-0060 IMPACT OF PRIOR TENOFOVIR DISOPROXIL FUMARATE (TDF) TREATMENT DURATION ON TENOFOVIR ALAFENAMIDE (TAF) SAFETY PROFILE IN VIRALLY SUPPRESSED CHRONIC HBV PATIENTS SWITCHED FROM TDF TO TAF}

${ }^{1}$ Henry Lik Yuen Chan*, ${ }^{2}$ Pietro Lampertico, ${ }^{3}$ Sang Hoon Ahn, ${ }^{4}$ Scott Fung, ${ }^{5}$ Ho Bae, ${ }^{6}$ Alnoor Ramji, ${ }^{7}$ Jung Sung Lee, ${ }^{8}$ Stephen Shafran, ${ }^{9}$ Stuart Gordon, ${ }^{10}$ Charles Phan, ${ }^{11}$ Susanna Tan, ${ }^{11}$ John Flaherty, ${ }^{11}$ Anuj Gaggar, ${ }^{11}$ George Wu, ${ }^{11}$ Vithika Suri, ${ }^{12}$ Daryl Lau, ${ }^{13}$ Kwan Soo Byun, ${ }^{14}$ Kosh Agarwal, ${ }^{15}$ Young-Suk Lim, ${ }^{16}$ Maria Buti. ${ }^{1}$ Institute of Digestive Disease, Department of Medicine and Therapeutics, and State Key Laboratory of Digestive Disease, The Chinese University of Hong Kong, Hong Kong; ${ }^{2}$ Fondazione IRCCS Ca' Granda Ospedale Maggiore Policlinico, Università degli Studi di Milano, Italy; ${ }^{3}$ Yonsei University College of Medicine, Korea, South; ${ }^{4}$ Toronto Centre for Liver Disease, Toronto General Hospital, University Health Network, Canada; ${ }^{5}$ Asian Pacific Liver Center, St. Vincent Medical Center, USA; ${ }^{6}$ The University of British Columbia, Canada; ${ }^{7}$ nje University College of Medicine, Korea, South; ${ }^{8}$ University of Alberta, Edmonton, Canada; ${ }^{9}$ Henry Ford Health System, USA; ${ }^{10}$ Greater Houston Gastroenterology, Sugar Land, USA; ${ }^{11}$ Gilead Sciences, Inc., USA; ${ }^{12}$ Beth Israel Deaconess Medical Center, Harvard Medical School, USA; ${ }^{13} \mathrm{Korea}$ University Guro Hospital, Korea, South, ${ }^{14}$ Institute of Liver Studies, King's College Hospital, UK; ${ }^{15}$ Asan Medical Center, University of UIsan College of Medicine, Korea, South; ${ }^{16}$ Centro de Investigación Biomédica en Red de Enfermedades Hepáticas y Digestivas, Hospital Universitari Valle Hebron, Spain

\subsection{6/gutjnl-2020-IDDF.143}

Background TAF demonstrated noninferior efficacy to TDF with a superior bone and renal safety profile, in viremic chronic HBV (CHB) patients through week 96, and in virally suppressed patients switched from TDF to TAF at 48 weeks. The duration of prior TDF therapy may influence the degree and rate of recovery of bone and renal function following switch to TAF. Here, we evaluate the impact of prior TDF treatment duration on the safety profile of TAF in virally suppressed patients.

Methods In a double-blind, randomized, multicenter, activecontrolled, Phase 3 study, $488 \mathrm{CHB}$ patients who were virologically suppressed on TDF for 1 year, and on TDF monotherapy for 6 months were randomized $1: 1$ to switch to TAF or continue TDF for 48 weeks. In patients originally randomized to receive $\mathrm{TAF}$, renal, bone, and lipid parameters were categorically evaluated by the duration of prior TDF treatment:

Results Of the 243 patients switched to TAF from TDF, 105 (43\%) and 138 (57\%) had received TDF for 50 years, 74\% male, $80 \%$ Asian, median eGFR ${ }_{\mathrm{CG}} 91 \mathrm{~mL} / \mathrm{min}, 4 \%$ and $12 \%$ had osteoporosis at hip and spine, respectively. Similar changes in renal, bone, and lipid parameters following 48 weeks of TAF treatment were observed by TDF treatment duration. A 